Reprod. Nutr. Dévelop., 1980, 20 (4 B), 1181-1186.

\title{
Etude cinéradiologique quantitative de la motricité antrale
}

\author{
par Michèle BOIRON, C. GAUDEAU *, P. ROULEAU **, J. THOUVENOT \\ Laboratoire de Physiologie, U. E. R. Médecine, B. P. 3223, 37032 Tours Cedex, France \\ * Laboratoire de Bioinformatique, U. E. R. Médecine, Tours \\ ** Service de Radiologie B, C. H. U. Bretonneau, 37045 Tours Cedex
}

Summary. Quantifative study of antral motility from $X$-ray pictures.

The quantitative determination of antral motility from $X$-ray pictures was studied in 12 normal fasting subjects whose average age was 25 . A sequence of 10 pictures was obtained while the subjects were lying prone, in apnea during $30 \mathrm{sec}$, and after drinking a sulfate baryum suspension. The sinus and antral surfaces were quantitatively determined by a videoplanimeter. The antral area decreased from a relaxed state to 50 p. 100 in $10.4 \pm 2.8 \mathrm{sec}$ and completed emptying in $23.1 \pm 3.6 \mathrm{sec}$. The maximal surface included a portion of the sinus and was evaluated in a range of 5.5 to $12 \mathrm{~cm}^{2}$. The relaxed period was very short. A coefficient to evaluate the contractile efficiency of the antrum is given by the product of total surface variation per gastric period.

\section{Introduction.}

Si les mécanismes de l'évacuation gastrique sont connus dans leurs grandes lignes (Hunt et McDonald, 1954 ; Cooke, 1975), les données quantitatives manquent pour la définition des caractéristiques contractiles des trois compartiments impliqués : corps, antre et bulbe duodénal. Les procédés automatiques de traitement d'images apportent ici une méthodologie qui nous a déjà permis d'analyser la propagation des ondes contractiles du corps gastrique (Boiron et al., 1977). Cependant, le fonctionnement du segment antral dans l'évacuation gastrique a motivé la présente étude où il s'agit de définir des caractéristiques contractiles rapportées au temps dans les conditions de l'exploration fonctionnelle chez l'Homme sans avoir recours à une sonde toujours à l'origine de stimuli réflexogènes. Or, sur le plan physiologique, ces trois territoires en présence possèdent une certaine autonomie tant sur le plan électrophysiologique (Sarna et Daniel, 1974), qu'hormonal (Fara, Praissman et Berkowitz, 1979). Mais, le rôle de la distension pour un contenu donné mérite d'être précisé. L'intérêt pratique est de pouvoir analyser les troubles fonctionnels, par exemple lors d'ulcères gastro-duodénaux (Keet, 1957 ; Bracco, Giorgio ef Thouvenot, 1976 ; Donner, 1976). 


\section{Matériel et méthodes.}

Cette étude a porté sur 12 sujets volontaires ne présentant aucune affection gastroduodénale (moyenne d'âge: $25 \pm 3$ ans).

Nous avons travaillé sur des séries radiographiques obtenues avec amplificateur de luminance (ampliphotographie). Cet appareil délivre des radiographies de petit format $(7 \times 7 \mathrm{~cm})$ avec l'indication précise des temps de saisie. Les séries de 10 clichés espacés toutes les $3 \mathrm{sec}$ sont obtenues lorsque les sujets sont en procubitus, en oblique postérieure gauche.

Toutes les séries ont été effectuées dans les mêmes conditions : les sujets à jeun depuis la veille ingèrent, en position debout, $250 \mathrm{ml}$ de bouillie barytée, en une seule fois (sulfate de baryłe 80 p. 100 + excipient aromatisé Radiobaryx). Puis, après contrôle radioscopique de l'évacuation gastrique, ils sont basculés en procubitus. Les sujets sont en apnée pendant toute la durée de la séquence radiologique, soit $30 \mathrm{sec}$.

Les 10 clichés ampliphotographiques sont analysés pour la quantification de la surface antrale. A cette fin, on détermine d'une part, les contours supérieur et inférieur, et d'autre part les points minimums entre ces deux contours. Ces points sont reliés pour la fermeture de la chambre antrale, ce qui définit ainsi la surface à mesurer (fig. 1). Puis, intervient l'étape de planimétrie au vidéoplanimètre SIEA (Société d'Informatique, d'Electronique et d'Automatique) (Lafargue, 1978).

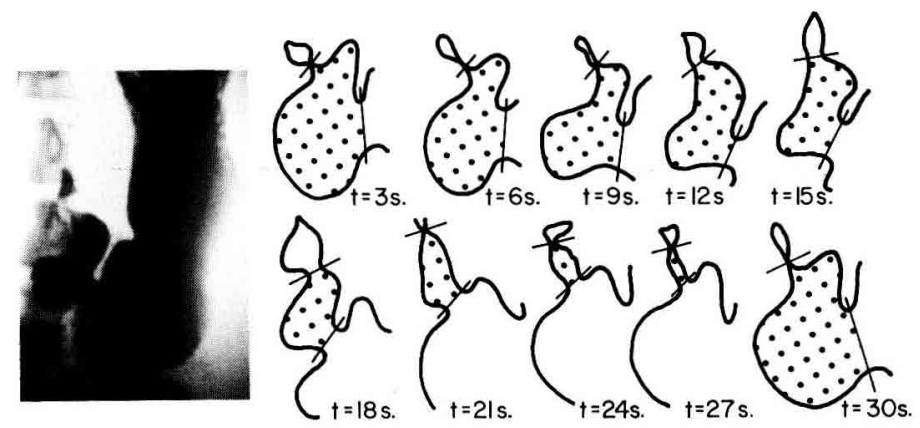

FIG. 1. - A gauche : Un des 10 clichés radiologiques d'une séquence de $30 \mathrm{sec}$. A droite : Les contours successifs (temps en sec) : les contours supérieur ef inférieur sont fermés au point où la distance intercontour est minimale.

Le vidéoplanimètre (fig. 2) permet de mesurer la surface délimitée par un contour fermé. Pour cela, le contour est établi à l'aide d'un feutre noir, sur un calque

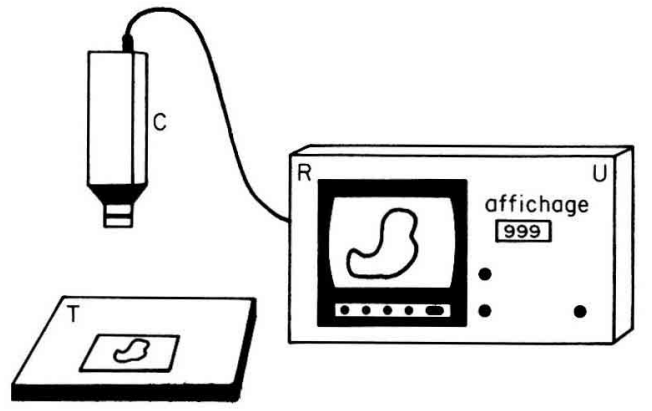

FIG. 2. - Le Vidéoplanimètre S. I. E. A. A gauche : la caméra de télévision (C) avec la fable lumineuse (T) ; A droite : l'unité de comptage $(U)$ et de visualisation du contour sur écran (R). 
directement placé au contact de lo radiographie sur la table lumineuse horizontale. Le principe de l'appareil est le suivant : pour chaque ligne, lorsque le balayage rencontre la première limite en entrant dans le domaine de la forme, il déclenche le comptage d'impulsions délivrées par une horloge $(5 \mathrm{MHz})$. Ce comptage est suspendu lorsque le faisceau d'analyse franchit la deuxième limite en sortant du domaine. Cette opération se reproduit de haut en bas pour les lignes successives ef les données du comptage sont cumulées.

\section{Résultats.}

Les variations planimétriques entre deux minimums de la surface antrale ont pour période $23,1 \pm 3,6 \mathrm{sec}(\mathrm{n}=7)$.

La cinétique de la contraction apparaît sur un diagramme surface-temps (fig. 3 ) : la courbe obtenue décroît globalement de manière sigmoïde à partir d'un maximum

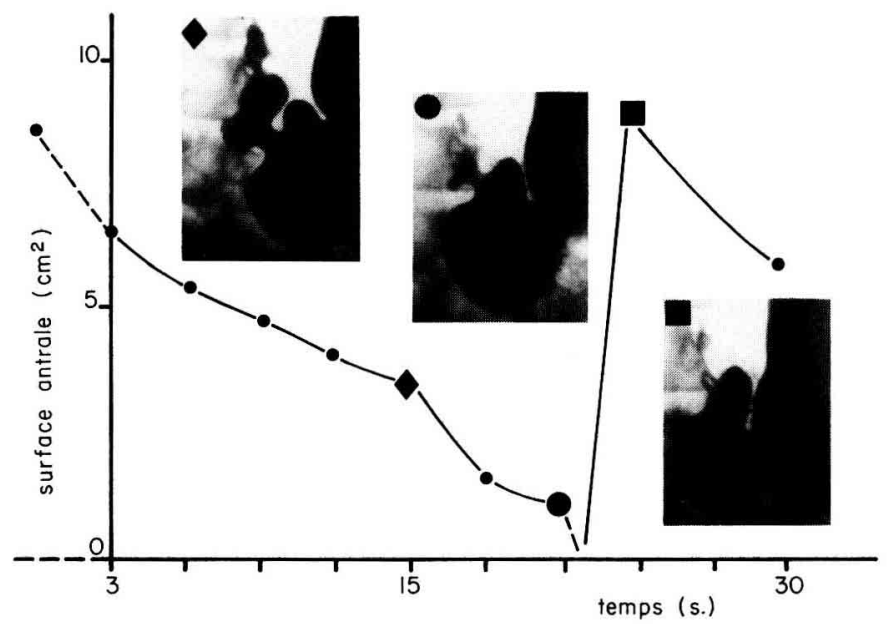

FIG. 3. - Evolution (temps en sec) de la surface de projection plane $\left(\mathrm{cm}^{2}\right)$ de la région anirale délimitée par ses deux zones de striction. Au cours du cycle contractile de l'antre, la surface évolue entre le maximum (relâchement) et le minimum (contraction) en deux phases : l'une rapide (réduction de 50 p. 100 en $9 \mathrm{sec}$ ), l'autre plus lente (en $12 \mathrm{sec}$ ). Les 3 radiographies correspondent aux stades d'isolement antral $\bullet$, de contraction maximale $\bullet$, de réplétion générale incluant l'antre et la partie distale du sinus m.

$\left(5,5\right.$ à $12 \mathrm{~cm}^{2} ; 6,6 \mathrm{~cm}^{2}$ pour le cycle montré fig. 3$)$. Pour certaines $(n=4)$, ces courbes présentent deux phases : l'une rapide où la surface de projection décroît de 50 p. 100 en 10,4 $\pm 2,8 \mathrm{sec}$, l'autre légèrement plus lente jusqu'à l'expulsion complète en $12,7 \pm 3,8 \mathrm{sec}$. Dans les autres cas $(n=3)$ la courbe est sigmoïde ou presque linéaire. Dans la phase terminale du cycle, toutes les courbes montrent une évolution accélérée (fig. 4).

Du fait qu'une séquence ne dépasse pas $30 \mathrm{sec}$, la contraction antrale s'intègre dans la phase gastrique de manière variable. Au mieux, elle débute au moment où 
la surface est maximale, dans d'autres cas, elle commence à un instant quelconque du cycle gastrique. Aussi, cela nous conduit à reconstituer le cycle antral à partir d'informations prises sur le cycle précédent ou suivant. Cette reconstitution apparaît en tirets, pour chaque sujet, sur la figure 4.

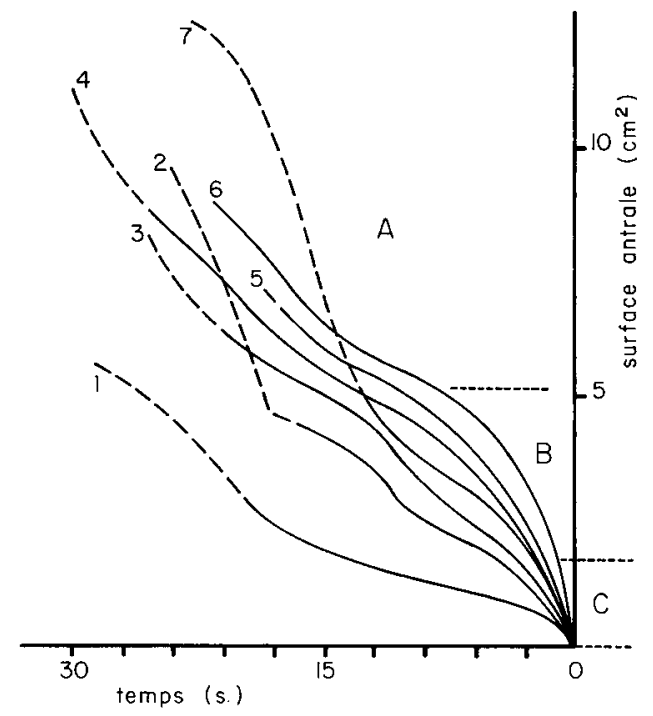

FIG. 4. - Comparaison de 7 séquences superposées d'évolufion antrale, obtenues dans les mêmes conditions chez 7 sujets différents (temps en sec sur compte à rebours à partir du minimum). L'évolution en deux phases montrée figure 3 est ici plus nuancée. Le tracé extrapolé à partir des données du cycle suivant ou précédent est représenté en tirets. Phase $A$ : évacuation rapide initiale $(1,2,6,7)$; Phase B : ralentissement intermédiaire ; Phase $C$ : accélération terminale.

Lorsque l'onde sous-cardiale commence sa progression, l'onde péristaltique précédente atteint le sinus au moment où l'antre se relâche. On a ainsi immédiatement après le minimum de surface, traduisant l'évacuation antrale maximale, un stade de réplétion général. A ce stade, le compartiment est limité en amont par la contraction du sinus et en aval par le pylore. L'anse canalaire proximale n'est pas encore entrée en activité contractile. La surface mesurée correspond alors à ce vaste compartiment qui comprend l'antre ef la partie distale du sinus. Cette zone forme chambre de mélange pendant 3 à $4 \mathrm{sec}$.

Au moment où l'anse canalaire proximale est atteinte, il se constitue la segmentation, à section limitée, entre le sinus et l'antre. Cette contraction peut isoler complètement ces deux compartiments et, dans ce cas, l'évacuation de l'antre est complète vers le bulbe duodénal. Dans d'autres cas (contraction incomplète), il y a reflux vers le sinus. L'évaluation de la contractilité peut se fonder sur le produit de 2 termes : variation totale de surface, comme approximation du volume et période de la contraction antrale. Ainsi, les cycles étudiés se classent de 126 à $309 \mathrm{~cm}^{2} \times \mathrm{s}$. 


\section{Discussion.}

La cinétique globale de la contraction antrale à pylore ouvert apparaît ici comme information d'un processus volumétrique et peut être confrontée aux données biomécaniques et manométriques connues (Stadaas et Schrumpf, 1974 ; Couturier, Rozé et Debray, 1972 ; Rees, Go et Malagelada, 1979). C'est ainsi que la cinétique en deux phases: l'une à variation de volume rapide ef l'autre légèrement plus lente, peut être rapprochée de celle décrite par Werle et al. (1940) chez le Chien : dans la première phase, la contraction antrale s'effectue à faible gradient de pression antrobulbaire, done à basse résistance; dans l'autre phase au contraire, la résistance s'accroit par contraction circulaire de l'antre terminal en fin de cycle.

II se pose maintenant le problème des relations avec le corps gastrique. Dans une conception compartimentale globale, Barker, Cobden et Axon (1979) étudient, grâce au marquage isotopique du contour, ce problème de la séparation des deux compartiments : estomac proximal et antre. Les volumes respectifs peuvent évoluer le plus souvent dans le même sens ou parfois en sens inverse lors des rétropulsions (Kelly, 1974). Or, d'après nos données, la jonction corps-antre met en jeu un territoire intermédiaire qui apparaît s'intégrer tantôt au corps, tantôt à l'antre : cette zone tampon a été individualisée anatomiquement depuis longtemps par Forsell (1913), zone qu'il appelle sinus.

Ainsi, ce système sinus-antre se comporfe comme un appareil de mélange et d'évacuation.

Journées Ingestion-Digestion-Absorption de l'Association française de Nutrition, Poris, 15-16 novembre 1979.

Remerciements. - Travail réalisé avec l'aide du CNRS el de la DRET.

\section{Références}

BARKER M. C. J., COBDEN I., AXON A. T. R., 1979. Proximal stomach and antrum in stomach emptying. Gut, 20, 309-311.

BOIRON M., GAUDEAU C., LONGEVIALLE C., BOUABDALLAH M., KURC-CARPENTIER C., THOUVENOT J., 1977. Analyse automatique des radiographies du système digestif par capteur optique. Path. Biol, 25, 215-224.

BRACCO F., GIORGIO I., THOUVENOT J., 1976. Interactions biomécaniques antroduodénales chez l'Homme en période interdigestive. Etude manométrique et endoscopique. J. Physiol., 72, $17 \mathrm{~A}$.

COOKE A. R., 1975. Control of gastric emptying and motility. Gastroenterology, 68, 804-816.

COUTURIER D., ROZE C., DEBRAY C., 1972 . Motor activity of the duodenum in man : Correlation with antral contraction. Digestion, 6, 1-8.

DONNER M. W., 1976. Normal and abnormal motility of the stomach. Radiol. Clin. North America, 14, 441-460.

FARA J. W., PRAISSMAN M., BERKOWITZ J. M., 1979. Interaction between gastrin, CCK and secretin on canine antral smooth muscle in vifro. Amer. J. Physiol., 236, E39-44.

FORSELL G., 1913. Uber die Beziehung der Rönfgenbilder des menschliden magens zu seinem anatomischen Bau. Beitrage zur anatomie und physiologie des Magens. Fortschr. A. D. Geb. Rig. Strahlen, suppl. 30, 1-42. 
HUNT J. N., MCDONALD I., 1954. The influence of volume on gastric emptying. J. Physiol., 126, 459-474.

KEET A. D., 1957. The prepyloric contraction in the normal stomach. Acta radiol., 148, 413-424.

KELLY K. A., 1974. Gastric motility after gastric operations. Surg. Ann., 6, 103-123.

LAFARGUE M., 1978. Le vidéoplanimètre. Biomechanics, Sofia, 7, 52-57.

REES W. D. W., GO V. L. W., MALAGELADA J. R., 1979. Antroduodenal motor response to solidliquid and homogenized meals. Gastroenterology, 76, 1438-1442.

IARNA S. K., DANIEL E. E., 1974. Threshold curves and refractoriness properties of gastric relaxation oscillators. Amer. J. Physiol., 226, 749-755.

STADAAS J., SCHRUMPF E., 1974. Intragastric pressure/volume relationship and gastrin release after distention of separate parts of the stomach. Gastroenterology, 9, 781-785.

WERLE J. M., BRODY D. A., LIGON E. W., READ M. R., QUIGLEY J. P., 1940. The mechanics of gastric evacuation. Amer. J. Physiol., 131, 606-614. 